CUBO A Mathematical Journal

Vol.14, No 01 , (111-117). March 2012

\title{
More on Approximate Operators
}

\author{
PHILIP J. MAHER \\ Mathematics And Statistics Group, \\ Middlesex University, Hendon Campus, The Burrough, \\ London Nw4 4 Bt, United Kingdom. \\ email: p.maher@mdx.ac.uk \\ and \\ Mohammad Sal Moslehian \\ Department Of Pure Mathematics, \\ Centre Of Excellence In Analysis On Algebraic Structures, \\ (CEAAS), Ferdowsi University Of Mashhad, P.O. Box 1159, Mashhad 917r5, Iran. \\ email: moslehian@ferdowsi.um.ac.ir, moslehian@member. ams.org
}

\begin{abstract}
This note is a continuation of the work on $(p, \epsilon)$-approximate operators studied by Mirzavaziri, Miura and Moslehian. [4]. We investigate approximate partial isometries and approximate generalized inverses. We also prove that if $\mathrm{T}$ is an invertible contraction satisfying $\left\|T^{*} T-T\right\|<\epsilon<\frac{2}{3 \sqrt{3}}$. Then there exists a partial isometry $V$ such that $\|\mathrm{T}-\mathrm{V}\|<\mathrm{K} \in$ for $\mathrm{K}>0$.
\end{abstract}

\section{RESUMEN}

Esta trabajo es una continuación del trabajo sobre operadores $(p, \epsilon)$-aproximados estudiados por Mirzavaziri, Miura y Moslehian [4]. Investigamos isometrías parciales 
aproximadas e inversas aproximadas generalizadas. También probamos que si T es una contracción invertible que satisface $\left\|T^{*} \mathrm{~T}-\mathrm{T}\right\|<\epsilon<\frac{2}{3 \sqrt{3}}$ entonces existe una isometría parcial $\mathrm{V}$ tal que $\|\mathrm{T}-\mathrm{V}\|<\mathrm{K} \epsilon$ para $\mathrm{K}>0$.

Keywords and Phrases: Hilbert space; approximation; unitary; partial isometry; polar decomposition; $(p, \epsilon)$-approximate operator

2010 AMS Mathematics Subject Classification: Primary 47A55; secondary 39 B52.

\section{Introduction}

This note is a continuation of the work on $(p, \epsilon)$-approximate operators and operator approximation studied in [4]. Mirzavaziri et al investigated $(p, \epsilon)$-approximate (co) isometries and $(p, \epsilon)-$ approximate unitaries. For example, a $(p, \epsilon)$-approximate isometry is defined as an operator $\mathrm{T}$ in $\mathcal{L}(\mathrm{H})$ for which

$$
\left\|\left[T^{*} T-I\right] f\right\| \leq \epsilon\|f\|^{p}
$$

where $p$ is a real number and $\epsilon$ a fixed positive number. They also proved, for example, the following result on unitary approximation: if to each $0<\epsilon<1$ an operator $\mathrm{T}$ in $\mathcal{L}(\mathrm{H})$ satisfies $\left\|\mathrm{T}^{*} \mathrm{~T}-\mathrm{I}\right\| \leq \epsilon$ and $\left\|\mathrm{T}^{*}-\mathrm{I}\right\| \leq \epsilon$ there corresponds a unitary operator $\mathrm{U}$ such that $\|\mathrm{T}-\mathrm{U}\|<\epsilon$.

In section 2 we investigate approximate partial isometries and approximate generalized inverses. In section 3 we investigate operator approximation. We prove (Theorem 3.2 below) that an invertible contraction $\mathrm{T}$ satisfying $\left\|T^{*} \mathrm{~T}-\mathrm{T}\right\|<\epsilon<\frac{2}{3 \sqrt{3}}$ can be approximated by a partial isometry.

Recall that a contraction $\mathrm{T}$ in $\mathcal{L}(\mathrm{H})$ is an operator such that $\|\mathrm{T}\| \leq 1$. Recall that the polar decomposition of an operator $\mathrm{T}$ says that $\mathrm{T}$ can be expressed uniquely as $\mathrm{T}=\mathrm{U}|\mathrm{T}|$, provided $\mathrm{KerU}=\mathrm{Ker}|\mathrm{T}|$, where $\mathrm{U}$ is a partial isometry. By definition, a partial isometry $\mathrm{U}$ is a isometric on $(\mathrm{KerU})^{+}$; and $|\mathrm{T}|$ denotes the positive square root of $\mathrm{T}^{*} \mathrm{~T}$.

\section{Approximate Operators}

In (1.1) (the example of a ( $p, \epsilon)$-approximate isometry) there is no question of letting $\epsilon \rightarrow 0$; for otherwise, the subject would collapse into triviality. For fixed $\epsilon$ the upshot of this section is that the $(p, \epsilon)$-approximate operators considered here coincide with their ordinary (exact) counterparts provided $p \neq 1$. In the cases studied here the operator $T$ we are concerned with must satisfy an operator equation of the form

$$
\mathrm{F}\left(\mathrm{T}, \mathrm{T}^{*}, \mathrm{~T}^{-}\right)=0
$$


(where $\mathrm{T}^{-}$is a generalized inverse of $\mathrm{T}$ : see Example 2.2 below). Our results hinge on the following lemma.

Lemma 2.1. Let $\mathrm{p}$ be a real number such that $\mathrm{p} \neq 1$ and let $\epsilon>0$. If

$$
\left\|F\left(T, T^{*}, T^{-}\right)\right\| \leq \epsilon\|f\|^{p}
$$

then $\left\|\mathrm{F}\left(\mathrm{T}, \mathrm{T}^{*}, \mathrm{~T}^{-}\right)\right\|=0$.

Proof. In (2.1) substitute $r f$ for $f$ where $r>0$. Then, by the linearity of $\mathrm{T}$,

$$
\left\|F\left(T, T^{*}, T^{-}\right)\right\| \leq \epsilon r^{p-1}\|f\|^{p} .
$$

If $p<1$ so that $r^{p-1}=r^{-k}$ where $k>0$ then $\epsilon r^{p-1}\|f\|^{p}=\frac{\epsilon\|f\|^{p}}{r^{k}} \rightarrow 0$ as $r \rightarrow \infty$. If $p>1$ then $\epsilon r^{p-1}\|f\|^{p} \rightarrow 0$ as $r \rightarrow 0$.

Example 2.2. (Partial isometries). There is the following (equivalent) algebraic definition of a partial isometry: $\mathrm{T}$ is a partial isometry if $\mathrm{T}=\mathrm{T}^{*} \mathrm{~T}$ [3, Problem 127, Corollary 3]. Given a real number $p$ and $\epsilon>0$, a $(p, \epsilon)$-approximate partial isometry is an operator $T$ in $\mathbb{B}(H)$ for which

$$
\left\|\left[T^{*} T-T\right] f\right\| \leq\|f\|^{p} .
$$

Let $\mathrm{F}\left(\mathrm{T}, \mathrm{T}^{*}\right)=\mathrm{T}^{*} \mathrm{~T}-\mathrm{T}$; if $\mathrm{p} \neq 1$ then, by Lemma $2.1, \mathrm{~F}\left(\mathrm{~T}, \mathrm{~T}^{*}\right)=0$ i.e. $\mathrm{T}$ is an (exact) partial isometry.

Counterexample 2.3. This shows that the condition $p \neq 1$ in Lemma 2.1 cannot be dropped. Let

$$
T=\left[\begin{array}{ccc}
0 & 0 & \sqrt{\epsilon} \\
0 & \sqrt{\epsilon} & 0 \\
\sqrt{\epsilon} & 0 & 0
\end{array}\right] \in M_{3}(\mathbb{C}) .
$$

Then, for $1<\epsilon<2$,

$$
\left\|\left[T^{*} T-T\right] f\right\|=|\epsilon-1| \sqrt{\epsilon}\|f\|<\sqrt{\epsilon}\|f\|<\epsilon\|f\|
$$

yet $T$ is not a partial isometry since $\|T f\|=\sqrt{\epsilon}\|f\|$ for all $f$ in $H$.

A $(p, \epsilon)$-approximate normal partial isometry is an operator $T$ in $\mathbb{B}(H)$ for which

$$
\left\|\left[T^{*} T^{2}-T\right] f\right\| \leq \epsilon\|f\|^{p} \quad(a)
$$

and

$$
\left\|\left[T^{2} T^{*}-T\right] f\right\| \leq \epsilon\|f\|^{p}
$$


for given $\epsilon>0$ and a real number $p$. Let $\mathrm{F}_{1}\left(\mathrm{~T}, \mathrm{~T}^{*}\right)=\mathrm{T}^{*} \mathrm{~T}^{2}-\mathrm{T}$ and $\mathrm{F}_{2}\left(\mathrm{~T}, \mathrm{~T}^{*}\right)=\mathrm{T}^{2} \mathrm{~T}^{*}-\mathrm{T}$; then if $p \neq 1$ Lemma 2.1 applied to $F_{1}$ and $F_{2}$ yields (a) $T^{*} T^{2}=T$ and (b) $T^{2} T^{*}=T$. Therefore, from (a), $\mathrm{T}^{*} \mathrm{~T}^{2} \mathrm{~T}^{*}=\Pi^{*}$ and, from (b), $\mathrm{T}^{*} \mathrm{~T}^{2} \mathrm{~T}^{*}=\mathrm{T}^{*} \mathrm{~T}$. Thus, $\mathrm{T}$ is normal and hence by, say (a), $T^{*} \mathrm{~T}=\mathrm{T}$.

Example 2.4. (Generalized inverses). An operator $\mathrm{T}^{-}$is said to be a generalized inverse of the operator $T$ if $T^{-} T=I$. An operator $T$ in $\mathbb{B}(H)$ has a generalized inverse if and only if RanT is closed [7, p. 261]. For an operator $\mathrm{T}$, with closed range, its Moore - Penrose inverse $\mathrm{T}^{+}$has range $\operatorname{RanT}^{+}=(\operatorname{KerT})^{\perp}$ and satisfies

$$
\begin{aligned}
\mathrm{T}^{+} \mathrm{T} & =\mathrm{T} \\
\mathrm{T}^{+} \mathrm{T}^{+} & =\mathrm{T}^{+} \\
\left(\mathrm{T}^{+}\right)^{*} & =\mathrm{T}^{+} \\
\left(\mathrm{T}^{+} \mathrm{T}\right)^{*} & =\mathrm{T}^{+} \mathrm{T}
\end{aligned}
$$

$(\mathrm{MP})$

and, further, $\mathrm{T}^{+}$is uniquely determined by these properties. If an operator $\mathrm{T}^{-}$satisfies properties (i), (iii) [(i), (iv)] it will be called a (i), (iii) [(i), (iv)] inverse of T. A (p, $\epsilon$ )-approximate generalized inverse of $T$ is an operator $T^{-}$in $\mathbb{B}(H)$ for which

$$
\left\|\left[T^{-} \mathrm{T}-\mathrm{T}\right] \mathrm{f}\right\| \leq \epsilon\|f\|^{\mathrm{p}}
$$

for $\epsilon>0$ and real $p$. Let $F_{1}\left(T, T^{+}\right)=T^{+} T-T, F_{2}\left(T, T^{+}\right)=T^{+} T^{+}-T^{+}, F_{3}\left(T, T^{+}\right)=\left(T^{+}\right)^{*}-$ $T^{*}-T$ and $F_{4}\left(T, T^{+}\right)=\left(T^{+} T\right)^{*}-T^{+} T$; then a $(p, \epsilon)$-approximate Moore-Penrose inverse pf $T$ is an operator $\mathrm{T}^{+}$in $\mathbb{B}(\mathrm{H})$ for which

$$
\left\|F_{i}\left(T<T^{+}\right) f\right\| \leq \epsilon\|f\|^{p}
$$

for $i=1, \ldots, 4$ and for $\epsilon>0$ and real $p$. Let $F\left(T, T^{-}\right)=T^{-} T-T$; then if $p \neq 1$, by Lemma 2.1, $\mathrm{F}\left(\mathrm{T}, \mathrm{T}^{-}\right)=0$ i.e. $\mathrm{T}^{-}$is a (exact) generalized inverse of $\mathrm{T}$; and, for $\mathrm{p} \neq 1$, applying Lemma 2.1 successively to $F_{1}, F_{2}, F_{3}$ and $F_{4}$ yields $F_{1}=F_{2}=F_{3}=F_{4}=0$ i.e. $T^{+}$satisfies (MP) so that $T^{+}$is the (exact) Moore - Penrose inverse of $\mathrm{T}$.

Counterexample 2.5. Again, we cannot drop the condition $p \neq 1$ in Lemma 2.1. Take $T=\epsilon S$ where

$$
S=\left[\begin{array}{cc}
\frac{1}{2} & \frac{1}{2} \\
\frac{1}{2} & \frac{1}{2}
\end{array}\right]
$$

and $0<\epsilon \leq 1$. Let $T^{\prime}=T$. Then

$$
\begin{aligned}
\left\|\left[T^{\prime} T-T\right] f\right\| & =\left|\epsilon^{3}-\epsilon\right|\|S f\| \leq \epsilon\left|\epsilon^{2}-1\right|\|S\|\|f\| \\
& =\epsilon\left|\epsilon^{2}-1\right|\|f\| \leq \epsilon\|f\|
\end{aligned}
$$

yet $T^{\prime}=\epsilon\left[\begin{array}{cc}\frac{1}{2} & \frac{1}{2} \\ \frac{1}{2} & \frac{1}{2}\end{array}\right]$ is not a generalized inverse of $T$ (except, as can be verified, if $\epsilon=1$ ) for, e.g., if $\epsilon=\frac{1}{2}$ then $\pi^{\prime} \mathrm{T}=\frac{1}{4} \mathrm{~T}$. 
Does the algebraic structure of approximate operators mirror that of their exact counterparts? For approximate isometries the answer is "yes". The product of two (exact) isometries is an (exact) isometry. The same is true for approximate isometries.

Proposition 2.6. The product of two $(\mathrm{p}, \epsilon)$-approximate isometries is a $\left(\mathrm{p}, \epsilon^{\prime}\right)$-approximate isometry.

Proof. For $p \neq 1$, by Lemma 2.1 , a ( $p, \epsilon)$-approximate isometry is an (exact) isometry. Therefore, we need to prove this result in the case of $p=1$. Accordingly, let $T_{1}$ and $T_{2}$ be two approximate isometries such that

$$
\left\|\left[T_{1}^{*} T_{1}-\mathrm{I}\right] \mathrm{f}\right\| \leq \epsilon_{1}\|\mathrm{f}\| \quad \text { and } \quad\left\|\left[\mathrm{T}_{2}^{*} \mathrm{~T}_{2}-\mathrm{I}\right] \mathrm{f}\right\| \leq \epsilon_{2}\|\mathrm{f}\|
$$

for $\epsilon_{1}>0, \epsilon_{2}>0$ for all $f$ in $H$. Assertion: if $\left\|\left[T^{*} T-I\right] f\right\| \leq \epsilon\|f\|$ for $\epsilon>0$ and for all $f$ in $H$ then $\|\mathrm{T}\|^{2} \leq \epsilon+1$. Proof of assertion:

$$
\left\|T^{*} T f\right\|=\left\|\left[T^{*} T-I\right] f+f\right\| \leq\left\|\left[T^{*} T-I\right] f\right\|+\|f\| \leq(\epsilon+1)\|f\|
$$

whence the result $\|\mathrm{T}\|^{2}=\left\|\mathrm{T}^{*} \mathrm{~T}\right\| \leq \epsilon+1$ follows by taking supremum over unit vectors.

Now,

$$
\begin{aligned}
\left\|\left[\left(T_{1} T_{2}\right)^{*}\left(T_{1} T_{2}\right)-I\right] f\right\| & =\left\|\left[T_{2}^{*}\left(T_{1}^{*} T_{1}-I\right) T_{2}-I+T_{2}^{*} T_{2}\right] f\right\| \\
& \leq\left\|T_{2}^{*}\right\|\left\|\left[T_{1}^{*} T_{1}-I\right] T_{2} f\right\|+\left\|\left[T_{2}^{*} T_{2}-I\right] f\right\| \\
& \leq\left\|T_{2}^{*}\right\| \epsilon_{1}\left\|T_{2} f\right\|+\epsilon_{2}\|f\| \\
& \leq\left(\left(\epsilon_{2}+1\right) \epsilon_{1}+\epsilon_{2}\right)\|f\|=\left(\epsilon_{1}+\epsilon_{1} \epsilon_{2}+\epsilon_{2}\right)\|f\| .
\end{aligned}
$$

We cannot expect a similar result about product of approximate partial isometries since it is not true that the product of two (exact) partial isometries is an (exact) partial isometry.

\section{Approximating Contractions}

We need the following lemma.

Lemma 3.1. Let $\mathrm{T} \leq 0,\|\mathrm{~T}\| \leq 1$ and $\left\|\mathrm{T}^{3}-\mathrm{T}\right\|<\epsilon<\frac{2}{3 \sqrt{3}}$. Then there is a self-adjoint partial isometry $\mathrm{S}$ such that $\|\mathrm{T}-\mathrm{S}\|<\mathrm{K} \in$ for a certain constant $\mathrm{K}>0$.

Proof. The conditions $\mathrm{T} \leq 0,\|\mathrm{~T}\| \leq 1$ imply that $\operatorname{sp}(\mathrm{T}) \subseteq[-1,0]$. Let $\delta_{1}, \delta_{2}\left(\delta_{1}<\delta_{2}\right)$ be the solutions of polynomial equation $t^{3}-t=\epsilon$ in $[-1,0]$. Then $\left|t^{3}-t\right|<\epsilon$ for all $\operatorname{sp}(T)$, whence $\mathrm{t} \in \operatorname{sp}(\mathrm{T}) \subseteq\left[-1, \delta_{1}\right] \cup\left[\delta_{2}, 0\right]$. 


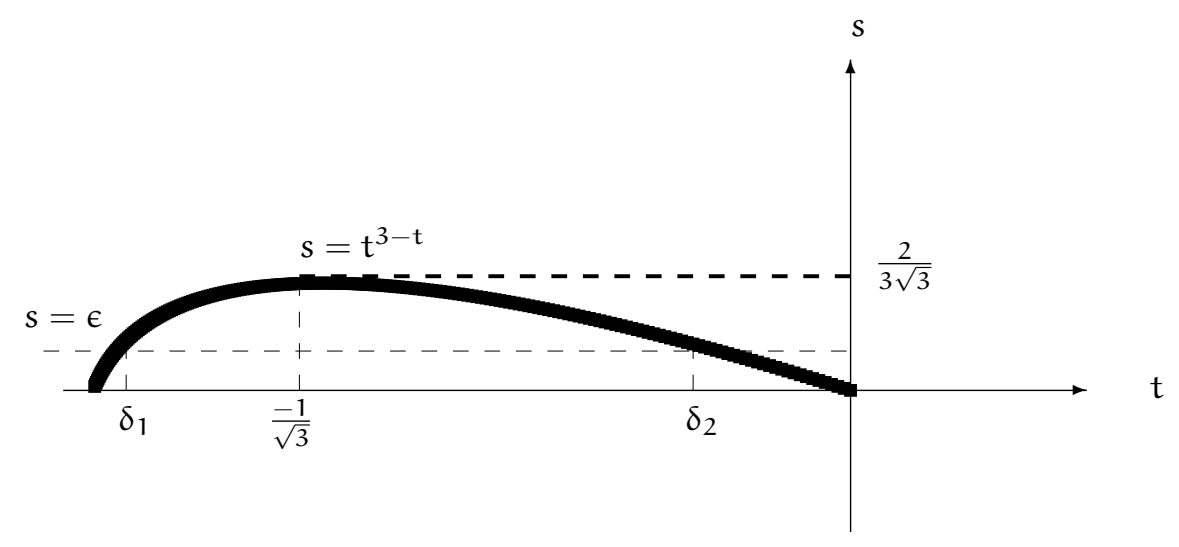

Therefore,

$$
\varphi(t)=\left\{\begin{array}{rl}
-1 & t \in\left[-1, \delta_{1}\right] \\
0 & t \in\left[\delta_{2}, 0\right]
\end{array}\right.
$$

is a continuous function on $\operatorname{sp}(T)$. Using the functional calculus, we observe that $S=\varphi(T)$ satisfies $\mathrm{S}^{*}=\mathrm{S}$ and $\mathrm{SS}^{*} \mathrm{~S}=\mathrm{S}$ and

$$
\|T-S\|=\sup _{t \in \operatorname{sp}(T)}|\varphi(t)-t|=\max \left\{1+\delta_{1},\left|\delta_{2}\right|\right\}<K \epsilon,
$$

for certain $K>0$.

Now we are ready to proof our next result.

Theorem 3.2. Let $\mathrm{T}$ be an invertible contraction and let $\left\|\mathrm{T}^{*} \mathrm{~T}-\mathrm{T}\right\|<\epsilon<\frac{2}{3 \sqrt{3}}$. Then there exists a partial isometry $\mathrm{V}$ such that $\|\mathrm{T}-\mathrm{V}\|<\mathrm{K} \in$ for a certain constant $\mathrm{K}>0$.

Proof. Let $\mathrm{T}=\mathrm{U}|\mathrm{T}|$ be the polar decomposition of $\mathrm{T}$. It is known that $\mathrm{U}$ is unitary, since $\mathrm{T}$ is invertible. Then

$$
\left\||\mathrm{T}|^{3}-|\mathrm{T}|\right\|=\left\|\mathrm{U}|\mathrm{T}| \mathrm{T}^{*} \mathrm{~T}-\mathrm{U}|\mathrm{T}|\right\|=\left\|\mathrm{T}{ }^{*} \mathrm{~T}-\mathrm{T}\right\|<\epsilon
$$

since the operator norm is unitarily invariant in the sense that $\|\mathrm{VXW}\|=\|\mathrm{X}\|$ for all arbitrary operators $\mathrm{X}$ and all unitaries $\mathrm{V}, \mathrm{W}$ in $\mathbb{B}(\mathcal{H})$. Utilizing Lemma 3.1 for $-|\mathrm{T}|$ we get a self-adjoint partial isometry $S$ such that $\||T|-S\|<K \in$ for a certain positive number $K$. Hence

$$
\|\mathrm{T}-\mathrm{US}\|=\|\mathrm{U}|\mathrm{T}|-\mathrm{US}\|=\|\mathrm{T} \mid-\mathrm{S}\|<\mathrm{K} \epsilon
$$

Since US(US)*US = US, the operator US turns into a partial isometry V.

If $\mathrm{T}$ acts on a finite dimensional Hilbert space $\mathcal{H}$, then the partial isometry $\mathrm{U}$ appeared in the polar decomposition of $\mathrm{T}$ is a unitary. So the proof of Theorem 3.2 above follows the following fact. 
Corollary 3.3. Let $A$ be an $m \times m$ contractive matrix such that $\left\|A A^{*} A-A\right\|<\epsilon<\frac{2}{3 \sqrt{3}}$. Then there exists a partial isometry $\mathrm{V}$ such that $\|\mathrm{A}-\mathrm{V}\|<\mathrm{K} \in$ for a certain constant $\mathrm{K}>0$.

Acknowledgement. The second author was supported by a grant from Ferdowsi University of Mashhad (No, MP89163MOS).

Received: June 2011. Revised: August 2011.

\section{References}

[1] S. Aaronson, Algorithms for Boolean function query properties, SIAM J. Comput. 32 (2003), no. $5,1140-1157$.

[2] P. R. Halmos, Positive approximants of operators, Indiana Univ. Math. J. 21, (1971/1972), pp. $951-960$

[3] P. R. Halmos, A Hilbert space problem book, $2^{\text {nd }}$ ed., Springer-Verlag, New York, (1982).

[4] M. Mirzavaziri, T. Miura and M. S. Moslehian, Approximate Unitaries in $\mathcal{B}(\mathcal{H})$. East J. Approx. 16 (2010), no. 2, 147-151.

[5] T. Miura, A. Uchiyama, H. Oka, G. Hirasawa, S. E. Takahasi and N. Niwa, A perturbation of normal operators on a Hilbert space, Nonlinear Funct. Anal. Appl. 13 (2008), no. 2, 291-297.

[6] G. J. Murphy, C*-algebras and Operator Theory, Academic press, Boston, 1990.

[7] A. E. Taylor and D. C. Lay, Introduction to functional analysis, $2^{\text {nd }}$ ed, John Wiley and Sons, New York, 1980. 